\title{
Temperature Dependence on Structural, Tribological, and Electrical Properties of Sputtered Conductive Carbon Thin Films
}

\author{
Yong Seob Park, ${ }^{*}$ Byungyou Hong, ${ }^{\dagger}$ Sang-Jin Cho,${ }^{\ddagger}$ and Jin-Hyo Boo ${ }^{\ddagger}, *$ \\ Department of Electrical and Electronic Engineering, Seonam University, Namwon 590-711, Korea \\ ${ }^{*}$ E-mail: sojujjang2@naver.com \\ ${ }^{\dagger}$ School of information and communication Engineering, Sungkyunkwan University, Suwon 440-476, Korea \\ ¿Department of Chemistry, Sungkyunkwan University, Suwon 440-746, Korea. *E-mail: jhboo@skku.edu \\ Received December 28, 2010, Accepted January 13, 2011
}

\begin{abstract}
Conductive carbon films were prepared at room temperature by unbalanced magnetron sputtering (UBMS) on silicon substrates using argon (Ar) gas, and the effects of post-annealing temperature on the structural, tribological, and electrical properties of carbon films were investigated. Films were annealed at temperatures ranging from $400{ }^{\circ} \mathrm{C}$ to $700{ }^{\circ} \mathrm{C}$ in increments of $100{ }^{\circ} \mathrm{C}$ using a rapid thermal annealing method by vacuum furnace in vacuum ambient. The increase of annealing temperature contributed to the increase of the ordering and formation of aromatic rings in the carbon film. Consequently, with increasing annealing temperature the tribological properties of sputtered carbon films are deteriorated while the resistivity of carbon films significantly decreased from $4.5 \times 10^{-3}$ to $1.0 \times 10^{-6} \Omega$-cm and carrier concentration as well as mobility increased, respectively. This behavior can be explained by the increase of $\mathrm{sp}^{2}$ bonding fraction and ordering $\mathrm{sp}^{2}$ clusters in the carbon networks caused by increasing annealing temperature.
\end{abstract}

Key Words : Conductive carbon film, Rapid thermal annealing, Magnetron sputtering, Tribological property, Carrier concentration and mobility

\section{Introduction}

Amorphous carbon (a-C) and hydrogenated amorphous carbon compounds $(\mathrm{a}-\mathrm{C}: \mathrm{H})$ have attracted attention due to their tribological and physical properties such as high hardness, low-friction coefficient, good wear resistance, chemical inertness, and optical transparency.$^{1-4}$ These films are used in a wide range of applications such as magnetic storage disks, cutting tools, and optical windows. Furthermore, carbon-based films show high thermal stability, which makes it possible to use them as protective coating materials in high temperature applications. However, these films have limited adhesion to substrates due to high intrinsic compressive stress. It is not easy to deposit carbon-based films with low compressive stress, high hardness, and high film thickness simultaneously; the usual deposition conditions for diamond-like carbon (DLC) films result in films with high intrinsic residual stress between the substrate and the film. The presence of large residual stresses leads to film failure and restricts the thickness of DLC films that will adhere to substrates. Residual stress can be minimized by annealing at a suitable temperature over a sufficiently long time..$^{5-7}$

In this study, an unbalanced magnetron sputtering (UBMS) method was used to prepare carbon films with a graphite target in $\mathrm{Ar}$ gas and room temperature conditions. The UBMS can raise the plasma density of the substrate though the selective strengthening of the magnetic field of the target ends, so that more of the secondary electrons can escape their confinement and effectively enhanced the sputtering rate and film density. Therefore, the use of UBMS technique is desirable to fabricate the hydrogen-free and dense conductive carbon films for the application to the electrodes in the electronic devices such as organic thin film transistor (OTFT), dye-sensitized solar cell (DSSC), and field emission display (FED). The synthesized carbon films were annealed at $400,500,600$, and $700^{\circ} \mathrm{C}$ by a vacuum furnace in vacuum ambient, and the effects of annealing temperature on structural, tribological, and electrical properties were investigated.

\section{Experimental}

The carbon films were deposited on a $2 \mathrm{~cm} \times 2 \mathrm{~cm}$ p-type (100) silicon substrate using an unbalanced magnetron sputtering (UBMS). The graphite (99.99\%) and high purity argon $(99.99 \%)$ were used as the sputtering target and the sputtering gas, respectively. Silicon substrates were cleaned using the usual RCA method and the background pressure of the process chamber was evacuated below $3 \times 10^{-4} \mathrm{~Pa}$ using diffusion pumps. The target power density was $20 \mathrm{~W} / \mathrm{cm}^{2}$, and the working pressure was $0.4 \mathrm{~Pa}$. Under these conditions, $150 \mathrm{~nm}$-thick carbon films were synthesized at room temperature with a $-200 \mathrm{~V}$ DC bias voltage. The fabricated films were annealed for $30 \mathrm{~min}\left(5^{\circ} \mathrm{C} / \mathrm{min}\right)$ at $400,500,600$, and $700{ }^{\circ} \mathrm{C}$ by using a vacuum furnace [Lenton 1200 Atmosphere Tube Furnace; LAH-12/300] in vacuum ambient.

Internal structure was characterized by Raman spectrometry [Jasco, MRS-300] and X-ray photoelectron spectroscopy [VG MICROTECH, ESCA-2000]. Surface morphology and Root mean square (Rms) surface roughness were observed using atomic force microscopy [Seiko, SPA-400]. 
Hardness was measured using a commercial nano-indentation instrument [Nano-indenter XP] with a continuous stiffness method (CSM) option. Friction coefficients were analyzed using a ball-on disk (BOD) tribometer in normal, dry ambient against a polished AISI 52100 steel ball with a diameter of $4.72 \mathrm{~mm}$ and sliding speed of $60 \mathrm{rpm}$. Film stress and the adhesion value were confirmed using a residual stress tester [J\&L Tech. JLCST022] and a nanoscratch tester [J\&L Tech. JLST022]. The electrical properties of annealed carbon films are investigated by Hall measurement [ECOPIA, HMS-3000].

\section{Results and Discussion}

Raman spectra of the carbon films annealed with increasing annealing temperature are shown in Figure 1(a). The G-peak position and the intensity ratio of the two peaks $\left(\mathrm{I}_{\mathrm{D}} /\right.$ $\mathrm{I}_{\mathrm{G}}$ ), observed by deconvoluting Raman spectra into two Gaussian curve fits in order to obtain quantitative information on $\mathrm{sp}^{3}$ content, are shown in Figure 2(b). From the Raman spectrum for as-deposited carbon film, the spectra show two broad peaks centered at $1548 \mathrm{~cm}^{-1}$ and $1376 \mathrm{~cm}^{-1}$, which are assigned to G- (graphite) and D- (disorder) modes, respectively. $G$ peak position in annealed carbon films moved to higher wavenumbers and the FWHM width in

(a)

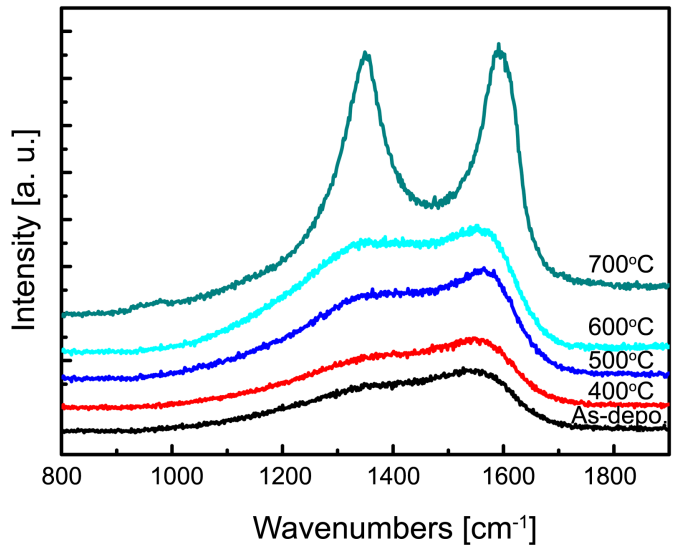

(b)

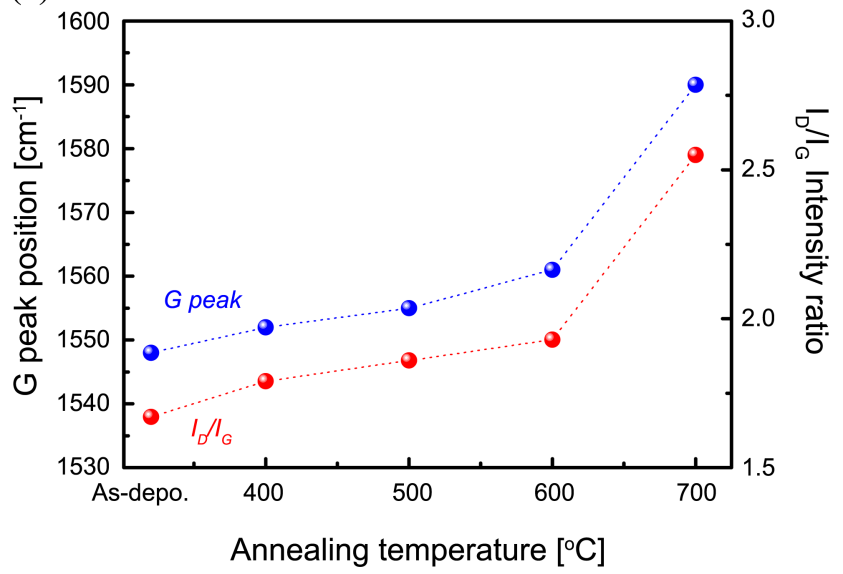

Figure 1. (a) Raman spectra of carbon films prepared at various annealing temperatures and (b) the variation in G-peak position and $\mathrm{I}_{\mathrm{D}} / \mathrm{I}_{\mathrm{G}}$ ratio as a function of annealing temperature.
$\mathrm{G}$ peak decreased with increasing annealing temperature, corresponding to an increase in the $\mathrm{sp}^{2}$ bonding contents. Also, $\mathrm{I}_{\mathrm{D}} / \mathrm{I}_{\mathrm{G}}$ is increased with increasing annealing temperature. In Raman spectra, D peak height should increase due to transformation of disordered rings to ordered six-fold rings and the $\mathrm{G}$ peak relates only to bond stretching of $\mathrm{sp}^{2}$ pairs, so it retains its intensity, and the $\mathrm{I}_{\mathrm{D}} / \mathrm{I}_{\mathrm{G}}$ ratio increases with increased ordering. ${ }^{8-10}$ Therefore, the increase of annealing temperature contributed to the increase of the ordering and formation of aromatic rings, indicating $\mathrm{sp}^{2}$ contents and $\mathrm{sp}^{2}$ clusters in the carbon film. At the temperature of 700 ${ }^{\circ} \mathrm{C}$, however, the $\mathrm{D}$ peak intensity and $\mathrm{I}_{\mathrm{D}} / \mathrm{I}_{\mathrm{G}}$ ratio increase sharply. These results are related to the graphitization of carbon films in response to removal of the disordering and to breakdown of the domain in $\mathrm{sp}^{2}$ clusters.

Figure 2(a) shows the C1s peak in XPS spectrum for the as-deposited carbon film and Figure 2(b) exhibits the variation in $\mathrm{sp}^{3} / \mathrm{sp}^{2}$ bonding ratio according to annealing temperature. The C1s XPS spectra were deconvoluted into two components with binding energies of $284.4 \mathrm{eV}\left(\mathrm{sp}^{2}\right.$ $\mathrm{C}=\mathrm{C})$ and $286.8 \mathrm{eV}\left(\mathrm{sp}^{3} \mathrm{C}-\mathrm{C}\right){ }^{10,11} \mathrm{The} \mathrm{sp}^{3} / \mathrm{sp}^{2}$ bonding ratio decreased with increasing annealing temperature. These results show the increase of $\mathrm{sp}^{2}$ components with increasing annealing temperature. ${ }^{10-12}$ Consequently, the increase of annealing temperature leads to greater ordering of the inner

(a)

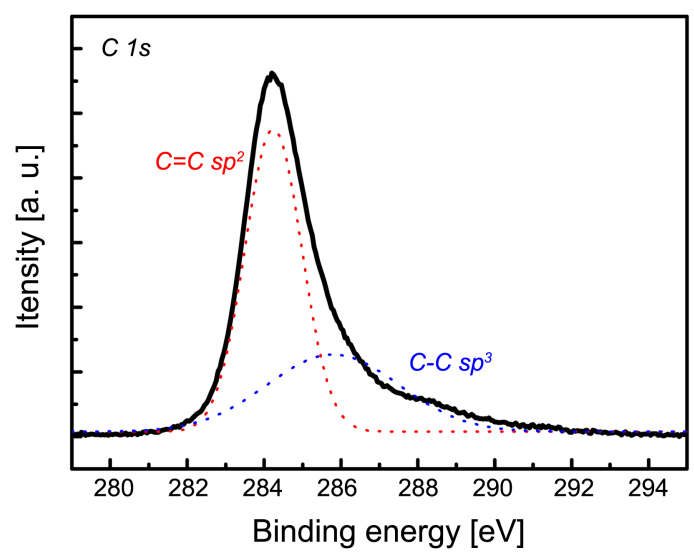

(b)

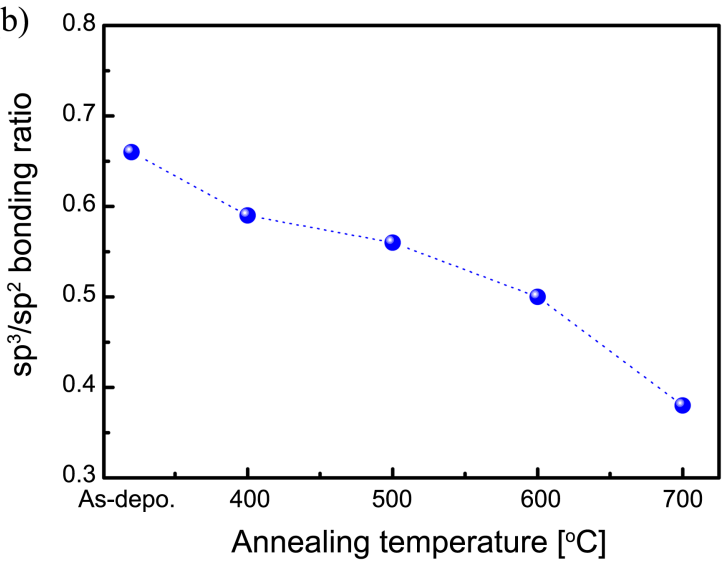

Figure 2. (a) The C1s peaks in XPS spectrum of as-deposited carbon films and (b) the variation in $\mathrm{sp}^{3} / \mathrm{sp}^{2}$ bonding ratio as a function of annealing temperature. 

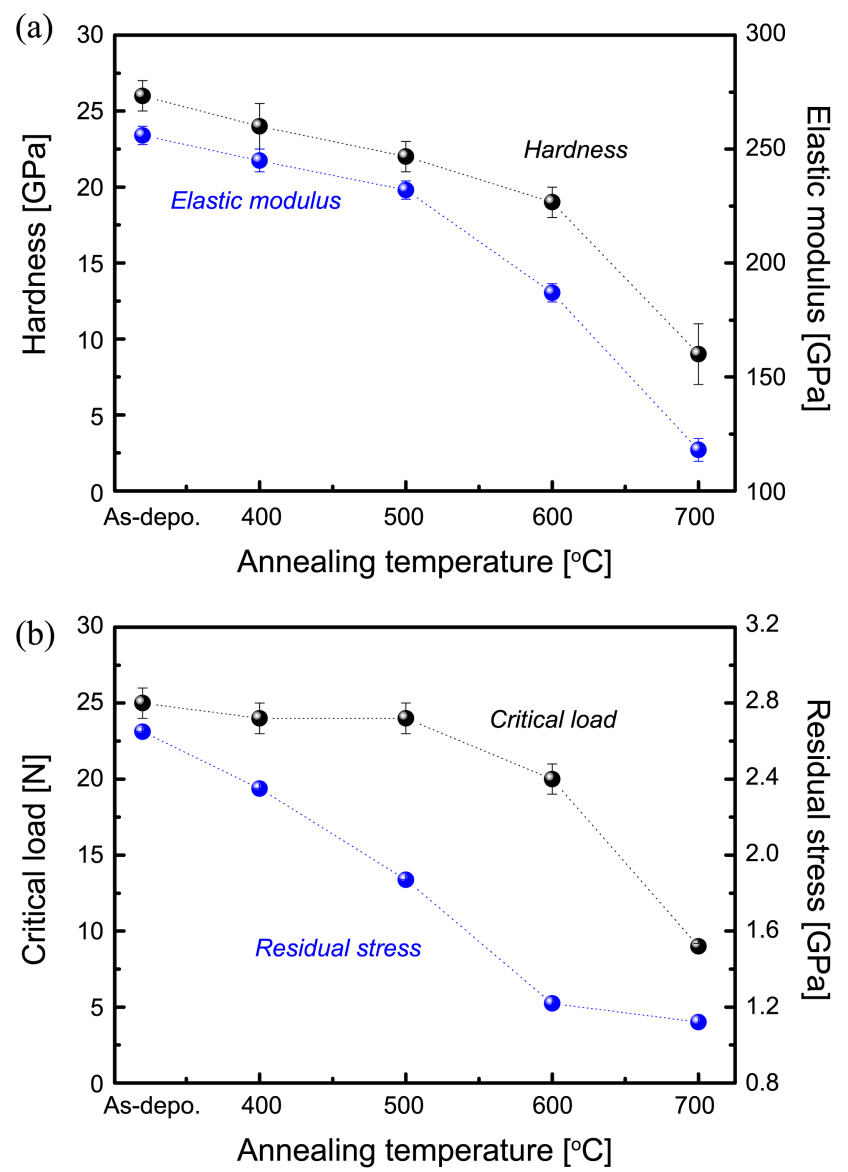

Figure 3. (a) Hardness and elastic modulus, and (b) residual stress and critical scratch load of carbon films as a function of annealing temperature.

structure of clusters in the carbon matrix.

In this study, the as-deposited carbon film prepared by UBMS method exhibited high hardness of about $25 \mathrm{GPa}$, resulting in a structure of cross-linked $\mathrm{sp}^{2}$ clusters caused by the bombardments of high energetic ions. ${ }^{4,13}$ This means that the hardness of carbon film is dependent on the degree of disorder cross-linked bonds in carbon network. As shown in Figure 3, however, the hardness and elastic modulus of carbon films are decreased with increasing annealing temperature, corresponding to an increase in the $\mathrm{sp}^{2}$ contents with the formation of aromatic rings, as revealed by Raman and XPS investigations. Specially, at the annealing temperature of $700{ }^{\circ} \mathrm{C}$, the hardness of carbon film is sharply decreased. These indicate that the increasing annealing temperature is associated with an the ordering of $\mathrm{sp}^{2}$ clusters in the film, and high temperature in $700{ }^{\circ} \mathrm{C}$ finally led to the change to a graphite-like structure. ${ }^{12,14,15}$ Also, the adhesion behavior of carbon films decreased with increasing annealing temperature, indicating an increase in ordered $\mathrm{sp}^{2}$ clusters in the carbon networks and a decrease in cross-linked bonds between the film and substrate., ${ }^{714}$ However, intrinsic stress gradually decreased with increasing annealing temperature. Thus, the increase of annealing temperature leads to structural relaxation related to ordering

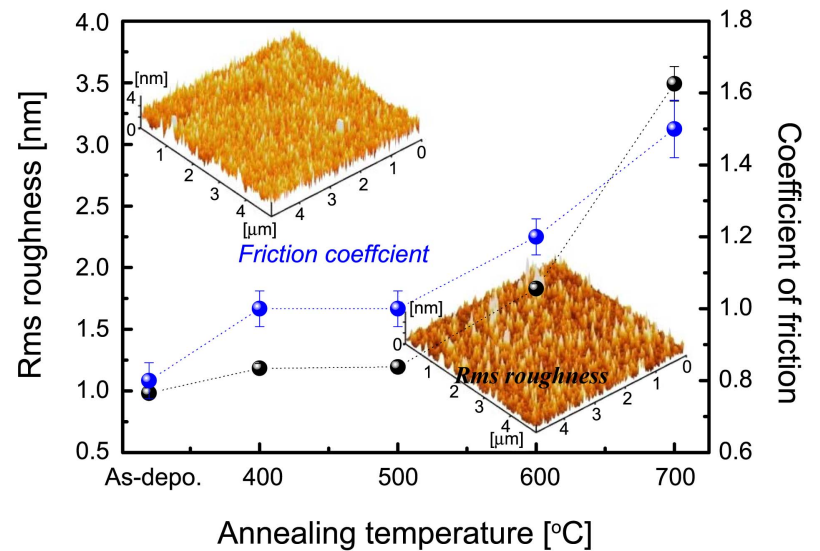

Figure 4. The variation in rms roughness and friction coefficient of carbon films prepared at different annealing temperatures with inserted AFM topography images of carbon films prepared at asdeposited and $700{ }^{\circ} \mathrm{C}$ annealing temperature.

in the $\mathrm{sp}^{2}$ clusters and is associated with a reduction of stress due to the decrease of defect density. ${ }^{14,15}$ Therefore, the increasing annealing temperature improves the intrinsic stress of the films, but leads to a decline in hardness and adhesion.

Figure 4 shows the variation in surface roughness and friction coefficient as a function of annealing temperature. The surface of the sputtered carbon films is ultra smooth due to the resputtering effect of energetic ions by negative DC bias voltage, and this would be considered to be related to the increase of $\mathrm{sp}^{2}$ bonding contents at the film surface. As shown in Figure 4, however, the increase of annealing temperature led to rougher surfaces and increased friction coefficients. The degradation in surface morphology and friction coefficient are associated with the clustering of $\mathrm{sp}^{2}$ carbon, ${ }^{16}$ as revealed by Raman and XPS investigations. Also, the rough surface and high friction coefficient in high annealing temperature are associated with the ordering $\mathrm{sp}^{2}$ clusters and the graphitization in carbon films. ${ }^{12}$ Consequently, the annealing temperature can affect the surface and density in film with the change of the film structure. ${ }^{12,14}$

Figure 5 shows the resistivity, carrier concentration, and

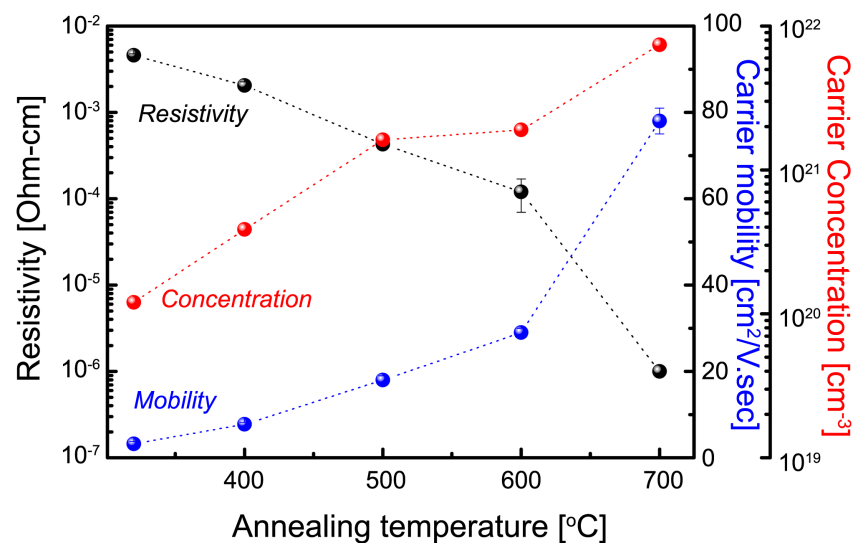

Figure 5. The variation of the resistivity, carrier concentration, and Hall mobility of carbon films as a function of annealing temperature. 
carrier mobility of carbon films as a function of annealing temperature. The as-deposited carbon film prepared at the low temperature showed the conductive properties such as low resistivity about $4 \times 10^{-3} \Omega \cdot \mathrm{cm}$, high carrier concentration about $1.2 \times 10^{20} \mathrm{~cm}^{-3}$, and high mobility about 3.2 $\mathrm{cm}^{2} / \mathrm{V} \cdot \mathrm{s}$. Also, it can be seen that the resistivity of carbon films significantly decreased from $4.5 \times 10^{-3}$ to $1.0 \times 10^{-6}$ $\Omega-\mathrm{cm}$ and carrier concentration and mobility increased with increasing annealing temperature, respectively. This behavior can be explained by the increase of $\mathrm{sp}^{2}$ bonding fraction and ordering $\mathrm{sp}^{2}$ clusters caused by increasing annealing temperature in the carbon networks. ${ }^{12}$ From the Raman results, the $\mathrm{G}$ peak for carbon films increases from vibrations of all $\mathrm{sp}^{2}$ sites and in both ring or chain configurations, however the existence of a D peak indicates the presence of aromatic rings and the intensity of $\mathrm{D}$ peak increases only from $\mathrm{sp}^{2}$ clusters in six fold aromatic rings. Therefore, the change in resistivity of carbon films with increasing annealing temperature can be understood by the increase of $\pi$ states associated with $\mathrm{sp}^{2}$ sites. Specially, low resistivity of carbon at $700{ }^{\circ} \mathrm{C}$ annealing temperature is associated with the graphitization of carbon film. The annealing temperature in carbon film can play an important role for the improvement of conductivity.

\section{Conclusions}

The carbon films were deposited on silicon substrate by UBMS method with a graphite target. The effect of annealing temperature on the tribological, structural and electrical properties of carbon films was investigated. The increase of annealing temperature is associated with the increases in ordered $\mathrm{sp}^{2}$ clusters and the $\mathrm{sp}^{2}$ bonding contents, and high annealing temperature is attributed to the graphitization of carbon films. Consequently, the increasing annealing temperature led to a decline in physical properties such as hardness, elastic modulus, adhesion, frication coefficient, and surface roughness. However, it is attributed to the improvement of the stress and conductivity in the film, reflecting that annealing of conductive carbon film will largely influence for the improvement of conductivity.

Acknowledgments. Support of this research by the National Research Foundation of Korea (Grant No: 20100025481) is gratefully acknowledged.

\section{References}

1. Liu, D.; Benstetter, G.; Lodermeier, E.; Akula, I.; Dudarchyk, I.; Liu, Y.; Ma, T. Surf. Coat. Technol. 2003, 172, 194.

2. Peng, X. L.; Baber, Z. H.; Clyne, T. W. Surf. Coat. Technol. 2001, $138,23$.

3. Sheeja, D.; Tay, B. K.; Lau, S. P.; Shi, X. Wear 2001, $249,433$.

4. Kulikorsky, V.; Bohc, P.; Franc, F.; Deineka, A.; Vorlicek, V.; Jastrabik, L. Diamond Relat. Mater. 2001, 10, 1076.

5. Li, H.; Xu, T.; Wang, C.; Chen, J.; Zhou, H.; Liu, H. Thin Solid Films 2006, 515, 2153.

6. Benlahsen, M.; Racine, B.; Clin, M.; Kellama, J. Non-Crystlline Solids 2000, 266-269, 783 .

7. Takadoum, J.; Rauch, J. Y.; Cattenot, J. M.; Martin, N. Surf. Coat. Technol. 2003, 174, 427.

8. Piazza, F.; Golanski, A.; Schulze, S.; Relihan, G. Appl. Phys. Lett. 2003, 82, 358

9. Ferrari, A. C.; Robertson, J. Phys. Rev. B 2000, 61, 14095.

10. Wang, X. C.; Li, Z. Q.; Wu, P.; Jiang, E. Y.; Bai, H. L. Appl. Surf. Sci. 2006, 253, 2087.

11. Jung, H.-S.; Park, H.-H. Thin Solid Films 2000, 377, 320.

12. Onoprienko, A. A.; Artamonor, V. V.; Yanchuk, I. B. Surf. Coat. Technol. 2003, 172, 189.

13. Onoprienko, A. A.; Yanchuk, I. B. Powder Metallurgy and Metal Ceramics 2006, 45, 190.

14. Conway, N. M. J.; Ferari, A. C.; Flewitt, A. J.; Robertson, J.; Milne, W. I.; Tagliaferro, A.; Beyer, W. Diamond Relat. Mater. 2006, 9, 765 .

15. Dillon, R. O.; Woolam, J. A. Physical Review B 1984, 29(6), 3482.

16. Tang, Z.; Zhang, Z. J.; Narumi, K.; Xu, Y.; Naramoto, H.; Nagai, S. J. Appl. Phys. 2001, 89, 1956. 